\title{
Dosaggio dell'ormone paratiroideo intatto (iPTH) con una metodica immunochemiluminometrica a due siti
}

\author{
F. Petrarulo, G. Tricarico, G. Pallotta, L. Gesualdo, \\ Unità Operativa di Nefrologia e Dialisi, Ospedale Civile - Altamura
}

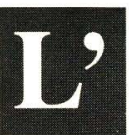

ormone paratiroideo (PTH), polipeptide costituito da 84 aminoacidi, è il principale fattore circolante che controlla la concentrazione del calcio extracellulare attraverso azioni sugli organi bersaglio con ritenzione del calcio da parte del rene e suo rilascio da parte dell'osso (Fig. 1).

Alla fine degli anni '60 l'applicazione delle metodiche radioimmunologiche (RIA) allo studio del PTH ci ha permesso di dosare la quantità di ormone circolante. Le prime metodiche radioimmunologiche utilizzate, comunque, sono risultate essere spesso imprecise, inconsistenti e non uniformi. È noto, infatti, che il PTH circolante è eterogeneo, si trova cioè sia come polipeptide intatto che come frammento (1).

La formazione dei frammenti, aminoterminale (PTH-NH2,1-34), carbossi-terminale (c-PTH, 53-84) e regione media (m-PTH, 28-48), è causata da modificazioni proteolitiche sia intra che extraghiandolari del polipeptide intatto. È stato dimostrato che il peptide circolante biologicamente attivo è il frammento amino-terminale, mentre i frammenti di PTH con terminale carbossilico sono biologicamente inerti $(2,3)$.

Divese metodiche radioimmunometriche sono state sviluppate per il dosaggio dei diversi frammenti. Queste metodiche sono risultate essere poco sensibili per il dosaggio di piccole quantità e poco affidabili per la possibilità di cross-reazione tra i vari frammenti. Inoltre, alcune metodiche radioimmunologiche, quali quelle che dosano il frammento della regione media ed il carbossi-terminale, non sempre riflettono l'attività biologica, in quanto i frammenti inattivi tendono ad accumularsi in corso di insufficienza renale.

L'introduzione delle metodiche radioimmunometriche (IRMA) per il dosaggio del PTH intatto ha apportato un notevole miglioramento in termini di riproducibilità e precisione allo studio dell'iperparatiroidismo primitivo e secondario. Infatti il dosaggio IRMA del PTTH riflette la secrezione del PTH in corso di ipo- ed ipercalcemia nel soggetto normale e nel dializzato. Pertanto, una comparazione significativa può essere fatta tra PTH e calcio indipendentemente dalla funzione renale. La metodica si basa sull'utilizzo di due anticorpi anti m-PTH e anti frammento amino-terminale che, formando un immunocomplesso con il PTH, permettono il dosaggio della molecola intatta $(4,5)$ (Tab. I).

Recentemente la marcatura dell'anticorpo anti frammento amino-terminale con una sostanza chemiluminescente, l'estere di acridinio (EA), ha aumentato la sensibilità dell'IRMA. Questa metodica chemiluminescente, denominata ICMA,

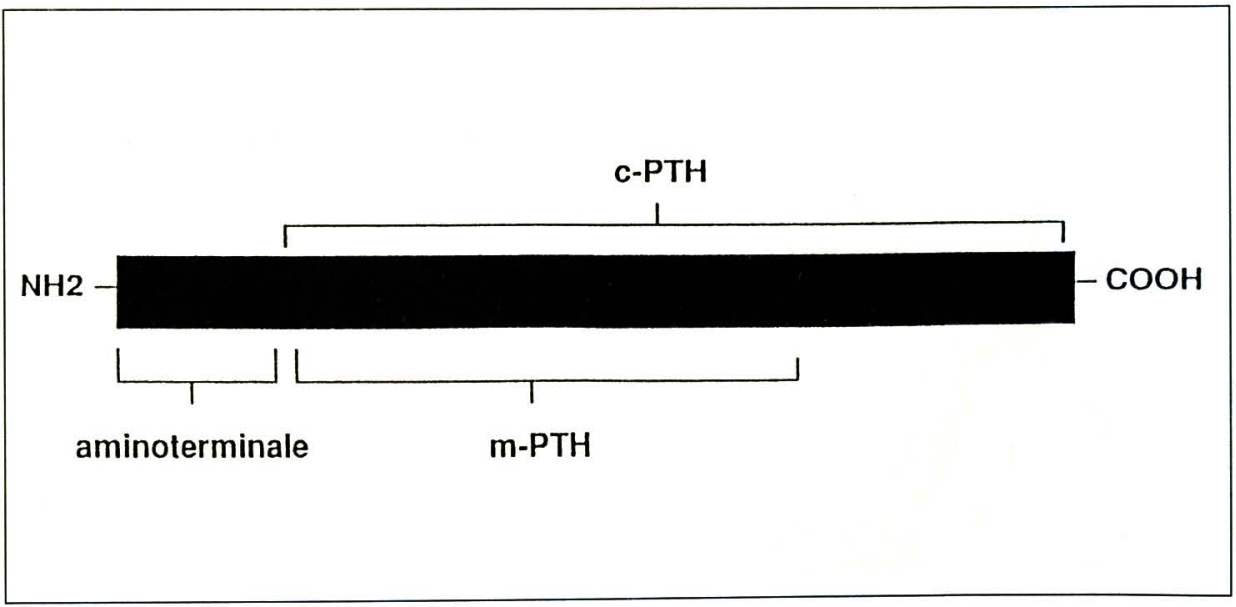

Fig. 1 - Ormone Paratiroideo (PTH): molecola intera e frammenti (aminoterminale NH2, intermedio MM, carbossiterminale $\mathrm{COOH}$ ). 
TAB. I - METODICHE RIA PER IL PTH

\begin{tabular}{ll}
\hline Anni '60 & $\begin{array}{l}\text { Applicazione delle metodiche radioimmunologiche (RIA) allo studio } \\
\text { del PTH }\end{array}$ \\
Limiti RIA & $\begin{array}{l}\text { : Poco sensibili per il dosaggio di piccole quantità e poco affidabili per la } \\
\text { possibilità di cross-reazione tra i vari frammenti }\end{array}$ \\
Anni '80 & : Metodiche radioimmunometriche (IRMA) \\
Metodo & $:$ due anticorpi - mPTH
\end{tabular}

non richiede l'uso di sostanze radioattive (Tab. II).

Ad oggi, la validità del metodo ICMA è stata riportata in pochi lavori $(6,7)$.

Scopo del nostro studio è stato quello di valutare la specificità, la sensibilità e l'accuratezza di questa metodica di immunochemiluminescenza e di verificare la sua utilità clinica in pazienti con insufficienza renale terminale.

\section{Casistica e metodi}

Sono stati studiati 70 soggetti normali e 60 pazienti in RDT (54 emodializzati e 6 pazienti in dialisi peritoneale) (Tab. III). I pazienti in emodialisi periodica ricevevano un trattamento dialitico standard trisettimanale variabile da 3 a 4 ore per singola seduta. Le superfici delle mem-

TAB. II - METODICA IN IMMUNOCHEMILUMINESCENZA A DUE SITI PER IL PTH

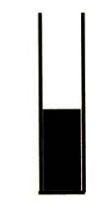

siero
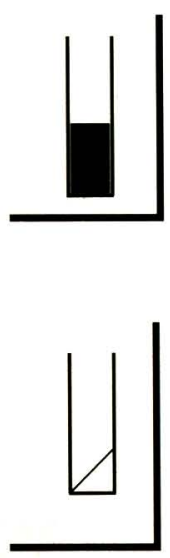

anticorpo di pecora anti-frammento aminoterminale (1-34) del PTH umano marcato con Estere di Acridinio

$+$

anticorpo di topo anti-regione 44-68 del PTH umano legato covalentemente a particelle paramagnetiche (Fase solida)

separazione libero-legato su lastra magnetica e decantazione del surnatante

aggiunta dei due reagenti necessari per la reazione chemiluminometrica e lettura all'analizzatore (Ciba Corning) brane utilizzate avevano un'area oscillante tra 1.0 e $1.6 \mathrm{~m}^{2}$ a seconda delle condizioni cliniche del paziente. Le concentrazioni del dialisato erano le seguenti: bicarbonato $39 \mathrm{mEq} / \mathrm{L}$, sodio 143 $\mathrm{mEq} / \mathrm{L}$, potassio $2.5 \mathrm{mEq} / \mathrm{L}$, calcio 3.5 $\mathrm{mEq} / \mathrm{L}$, magnesio $0.5 \mathrm{mEq} / \mathrm{L}$ ed assenza di glucosio.

Il PTH intatto è stato dosato con la metodica ICMA in tutti i soggetti esaminati utilizzando il Magic Lite PTH Kit (Ciba Corning Diagnostic, Milano). Questa metodica prevede l'utilizzo di due anticorpi, anti frammento (1-34) e anti frammento (44-68), marcati rispettivamente con l'estere di acridinio e con particelle paramagnetiche, che, formando un immunocomplesso con il PTH intatto, ne permettono il dosaggio. L'iPTH con metodica IRMA è stato dosato utilizzando l'Allegro Intact PTH Kit (Nichols Institute Diagnostic, USA). Il cPTH è stato dosato con metodo RIA-MAT della Byk-Gulden basato sulla competizione tra il frammento c-terminale di PTH del siero e PTH sintetico marcato con I-125 che reagiscono con un anticorpo antiPTH bovino.

Inoltre in tutti i pazienti sono stati dosati: il calcio ionizzato, la fosfatasi alcalina ed ossea. Infine, in 20 pazienti in emodialisi periodica, scelti in modo random, il PTH intatto è stato dosato in pre- e post-dialisi sia con la metodica ICMA che IRMA. Ancora, in questi pazienti venivano dosati in pre- e post-dialisi la fosfatasi alcalina, la fosfatasi ossea, il calcio ionizzato e il cPTH.

L'affidabilità e la riproducibilità del metodo sono stati valutati con l'analisi dei coefficienti di variazione rispettivamente intra-assay e inter-assay. I risultati sono stati analizzati statisticamente utilizzando il t-test di Student e la regressione lineare semplice; ove appropriato, $\mathrm{i}$ dati sono stati espressi come media \pm DS \pm $\mathrm{ES}$, si è considerata significativa una $\mathrm{p}<$ 0.05 .

\section{Risultati}

La determinazione del PTH effettuata nei 70 soggetti apparentemente normali ha fornito un valore medio di $38.34 \pm$ $16.84 \mathrm{pg} / \mathrm{ml}$ con range di riferimento di $12-87 \mathrm{pg} / \mathrm{ml}$ mentre i valori dell' ${ }^{2} \mathrm{PTH}$ nei 54 pazienti in emodialisi erano compresi tra 3.99 e $1700.1 \mathrm{pg} / \mathrm{ml}(\mathrm{x}=327 \pm$ $60.57 \mathrm{pg} / \mathrm{ml}$ ) e nei sei pazienti in perito- 
TAB. III - CASISTICA

\section{Soggetti normali}

n. 70

Pazienti in RDT

n. 60 età anagrafica $45.4 \pm 15.3$

età anagrafica $58.4 \pm 14.3$

età dialitica $\quad 64.1 \pm 62.8$ neo-dialisi tra 16 e $1107 \mathrm{pg} / \mathrm{ml}(\mathrm{x}=$ $404.83 \pm 511.96 \mathrm{pg} / \mathrm{ml}$ ) (Fig. 2). Il dosaggio del cPTH non mostrava variazioni statisticamente significative tra i valori di pre-dialisi $(11.04 \pm 0.9 \mathrm{ng} / \mathrm{ml})$ e quelli di post-dialisi $(11.2 \pm 1.0 \mathrm{ng} / \mathrm{ml})$ (Fig. 3). La calcemia totale variava da valori di pre-dialisi di $8.86 \pm 0.89 \mathrm{mg} / \mathrm{dl}$ a valori di post-dialisi di $9.89 \pm 0.88$ $\mathrm{mg} / \mathrm{dl}$ con un ES di \pm 0.12 in entrambi i casi ed una significatività di $p<0.001$

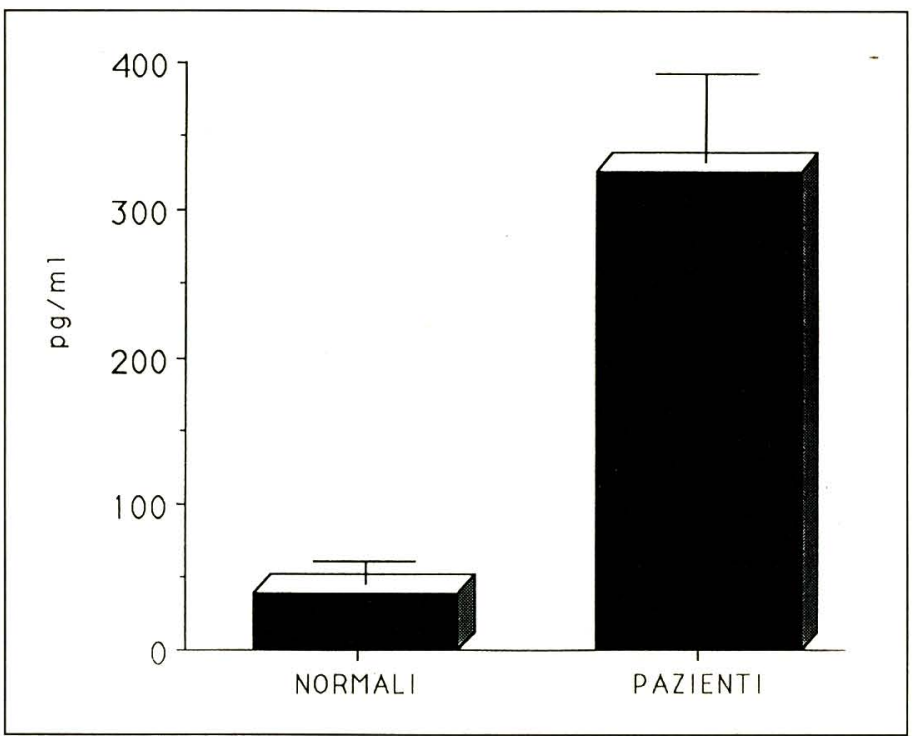

Fig. 2 - Dosaggio dell' iPTH con la metodica ICMA in soggetti normali e pazienti in dialisi.

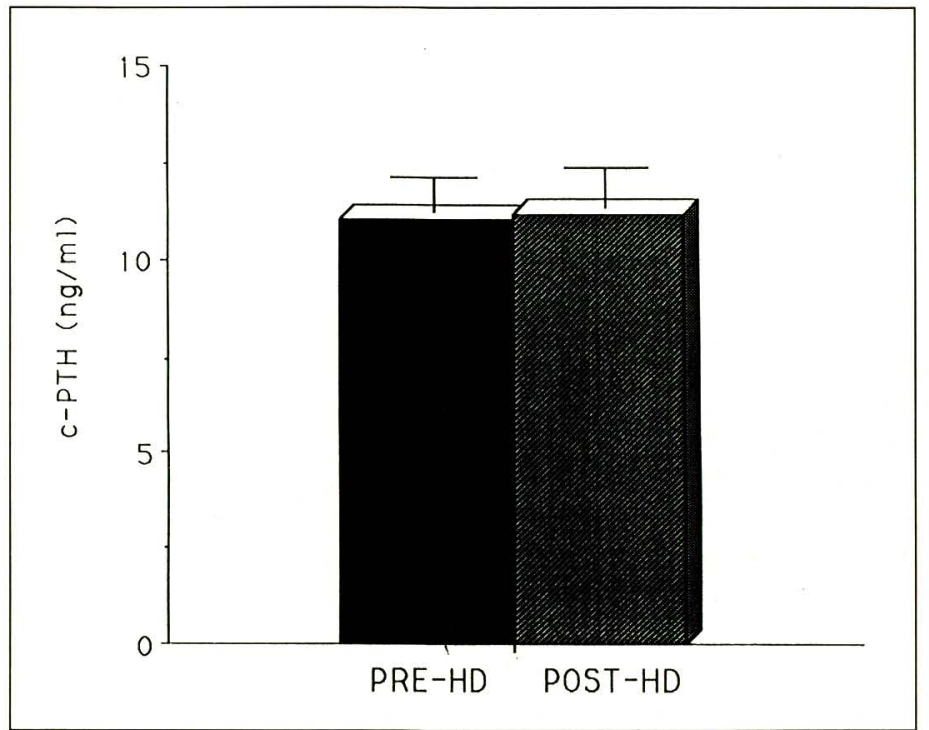

Fig. 3 - Dosaggio del c-PTH con metodica RIA ad inizio e fine seduta dialitica.

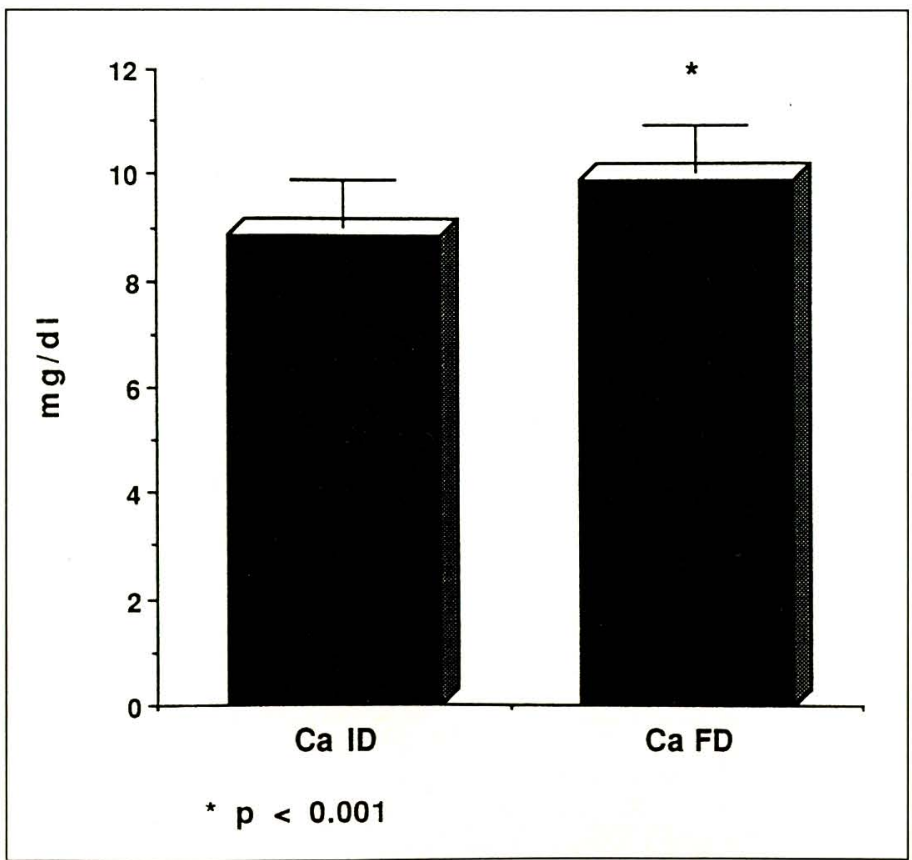

Fig. 4 - Variazioni del Ca totale ad inizio e fine seduta dialitica.

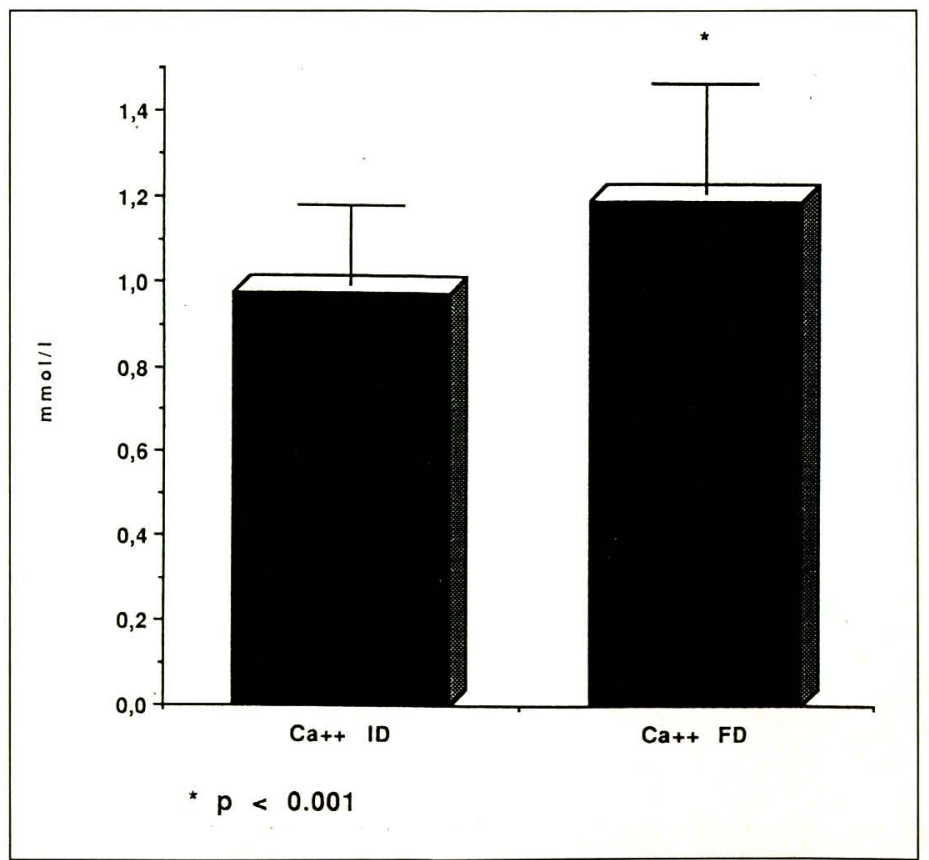

Fig. 5 - Variazioni del $\mathrm{Ca}^{++}$ad inizio e fine seduta dialitica. 


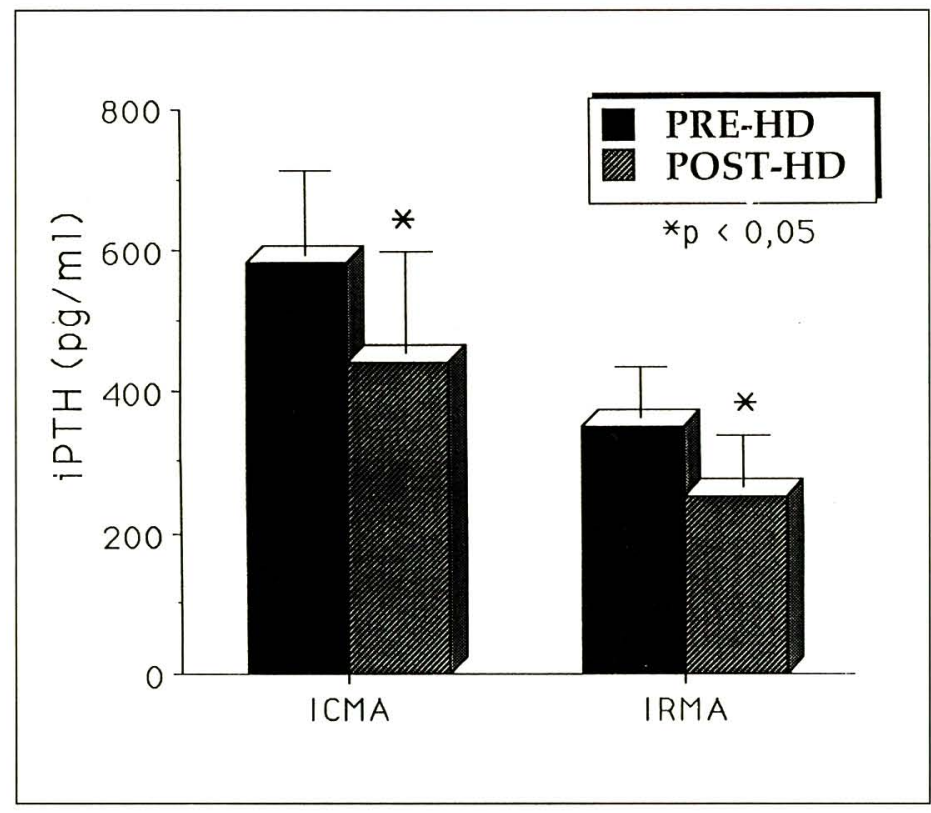

Fig. 6 - Valori dell'iPTH ad inizio e fine seduta dialitica con metodica ICMA ed IRMA.

(Fig. 4). La concentrazione plasmatica del calcio ionizzato evidenziava un significativo $(p<0.001)$ aumento nel postdialisi rispetto al pre-dialisi passando da $0.973 \pm 0.178 \mathrm{mmol} / \mathrm{L}$ a $1.196 \pm 0.256$ $\mathrm{mmol} / \mathrm{L}$ con un ES rispettivamente di \pm 0.025 e \pm 0.036 (Fig. 5) .

In concordanza con tale dato, abbiamo notato una riduzione statisticamente significativa $(\mathrm{p}<0.05)$ del dosaggio del iPTH nel post-dialisi rispetto al pre-dialisi sia con la metodica IRMA (pre-dialisi $351 \pm 71$, post-dialisi $252 \pm 74.5$ ) che con l'ICMA (pre-dialisi $583 \pm 120$, postdialisi $441 \pm 146$ ) (Fig. 6).

Inoltre si è evidenziata una correlazione significativa dei valori dell'iPTH fra le due metodiche (IRMA ed ICMA) sia in pre-dialisi $(\mathrm{r}=0.954)$ che in post-dialisi $(\mathrm{r}=0.989)$ (Figg. 7, 8).

I coefficienti di variazione intra-assay ed inter-assay erano rispettivamente $4.3 \pm$ 0.2 e $8.1 \pm 0.5$.

\section{Discussione}

L'iperparatiroidismo secondario rappresenta ancor oggi una delle complicanze più frequenti dell'uremia cronica terminale. Per la sua esatta definizione è di fondamentale importanza il dosaggio dell'ormone paratiroideo e dei suoi frammenti (8).

Tra le varie metodiche oggi in uso, quella immunochemiluminometrica, di recente introduzione, si è dimostrata, per alcuni aspetti, interessante. I nostri riscontri, confortati da quelli della letteratura $(6,7)$, hanno evidenziato, sia nei soggetti normali che in quelli in trattamento sostitutivo, precisione ed attendibilità, ulteriormente convalidati in situazioni di massima variabilità ionica ed ormonale quali quelle che si verificano durante il trattamento dialitico (9-12). In particolare l'iPTH dosato con metodica ICMA, al pari di quello dosato con metodica IRMA, ha mostrato una significativa riduzione in post-dialisi, contrariamente al cPTH i cui valori erano sovrapponibili in pre- e post-dialisi. La riduzione dell'iPTH nel post-dialisi, a differenza del comportamento del cPTH (frammento a scarsa attività biologica), conferma l'elevata attività biologica del frammento amino-terminale dell'ormone sensibile alla variazione del calcio ionizzato, come già evidenziato da diversi Autori (2).

I nostri dati, in accordo con quelli della letteratura (6), nel sottolineare la specificità, la sensibilità e l'accuratezza di questa nuova metodica, dimostrano che i risultati sia con l'IRMA che con l'ICMA sono sovrapponibili. L'ICMA, comunque, dovrebbe essere preferita perché meno rischiosa (non uso di sostanze radioattive) ed economicamente più vantaggiosa.

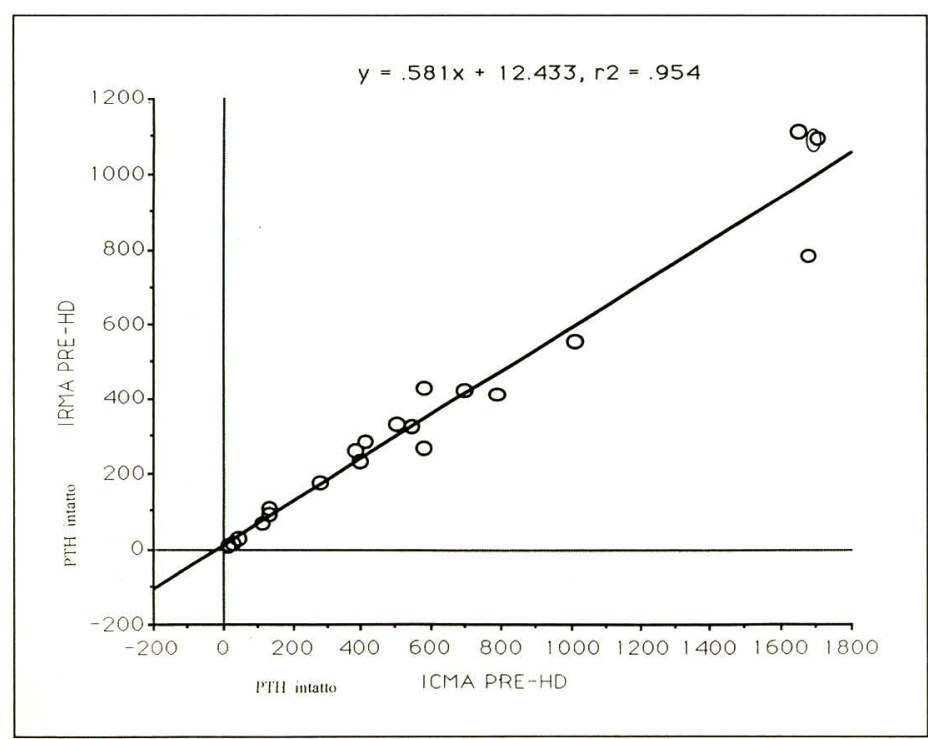

Fig. 7 - Correlazione tra $i$ valori di iPTH ad inizio dialisi con metodica ICMA ed IRMA.

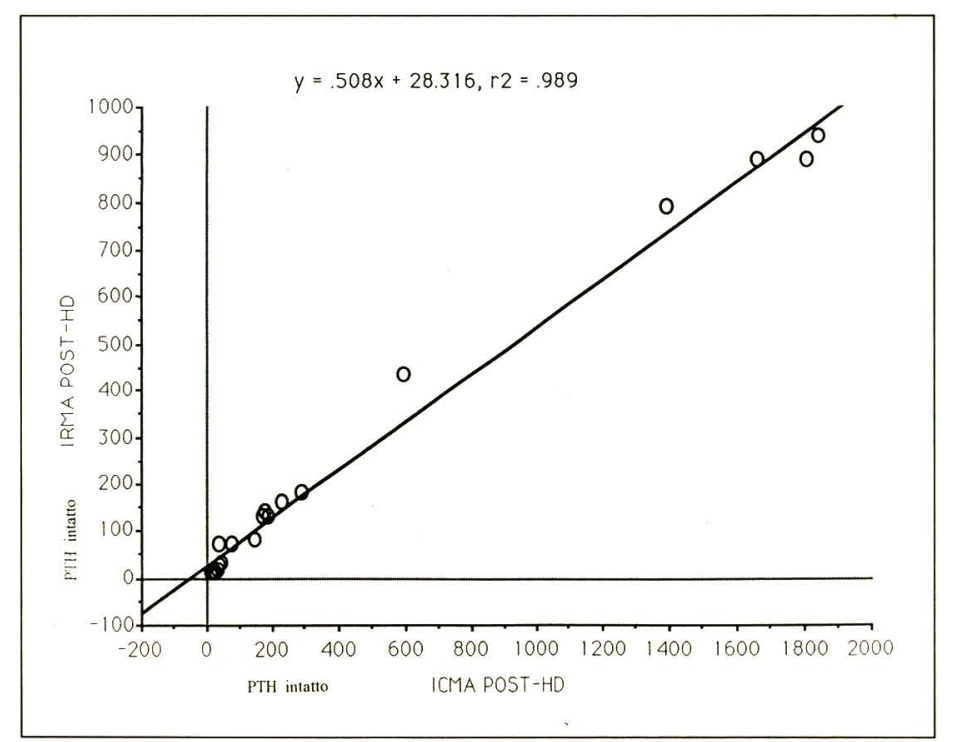

Fig. 8 - Correlazione tra $i$ valori di iPTH a fine dialisi con metodica ICMA ed IRMA. 


\section{BIBLIOGRAFIA}

1. Slatopolsky E, et al. Currents concept of the metabolism and radioimmunoassay of parathyroid hormone. J Lab Clin Med 1992; 99: 309.

2. Grassi C, et al. Studio epidemiologico-clinico in emodializzati per la valutazione di tre metodiche per la determinazione dei livelli plasmatici di paratormone. GIMM 1989; 4: 251-7.

3. Hackeng W, et al. Clinical implications of estimation of intact parathyroid hormone versus total immunireactive PTH in normal subjects and hyperparathyroid patients. J Clin Endocrinol Metab 1989; 63: 447.

4. Nussbaum S, et al. A highly sensitive two-site immunoradiometric assay of PTH and its clinical utility in evaluating patients with hypercalcemia. Clin Chem 1987; 33: 1364.

5. Bandini S, et al. Valutazione di una nuova metodica di dosaggio del PTH intatto nello studio della funzionalità dell'autotrapianto paratiroideo dopo paratiredectomia totale in emodializzati. GIMM 1989; 4: 251-7.

6. Morita A, et al. A two-site immunochemiluminometric assay for intact parathyroid hormone and its clinical utility in hemodialysis patients. Clin Nephrol, 1992; 3: 154-7.

7. Brown RC, et al. Circulating intact parathyroid hormone measured by a two-site immunochemiluminometric assay. J Clin Endocrinol Metab 1987; 65: 407.

8. Andress DL, et al. Comparison of parathyroid hormone assays with bone histomorphometry in renal osteodysytrophy. J Clin Endocrinol Metab 1986; 63: 1193.

9. Saha H., et al. Acute effect of dialysate calcium concentration and intravenous vitamine D3 on the secretion of parathyroid hormone in hemodialysis patients. Clin Nephrol, 1992; 3: 145-8.

10. D'Amour P, et al. IPTH values during hemodialisys: role of ionized $\mathrm{Ca}$, dialysis membranes and $\mathrm{iPTH}$ assay. Kidney Int, 1990; 38: 308-14.

11. Carley S.L. Ionized calcium concentration in maintenance hemodialysis patients. Clin Nephrol, 1992; 3: 167-70.

12. Massry SG, et al. Turnover of endogenous parathyroid hormone in uremic patients and those undergoing hemodialysis. Trans Am Soc Artif Intern Organs 1972; 18: 416-22. 